\title{
Influencia de las ráfagas de viento en la calidad de la energía de los parques eólicos
}

\section{(Influence of wind gusts in power quality on wind farms)}

\author{
Gonzalo Guerrón ${ }^{1}$, Edwin García ${ }^{1}$, Andrés Montero ${ }^{1}$
}

\begin{abstract}
Resumen:
La matriz energética mundial está cambiando debido a que el consumo de energía en los últimos años se ha duplicado. En muchos países la instalación de centrales de energías renovables no convencionales se ha convertido en la solución a este crecimiento. La integración de este tipo de energías a la red eléctrica causa interés en el estudio de las propiedades eléctricas que afectan la producción de energía. Este documento es una revisión de los conceptos y fenómenos de calidad de energía y cómo estos se generan en parques eólicos. La eficiencia de los aerogeneradores se ve afectada por diversos factores, entre los cuales se destacan: las maniobras en subestaciones y los factores climáticos como las ráfagas de viento causantes de variaciones en la curva de potencia. El monitoreo constante en los sistemas eléctricos y el análisis de datos ofrece un alto potencial en la mejora de los procesos de producción y distribución de la energía.
\end{abstract}

Palabras clave: Calidad de Energía; Parques Eólicos; Ráfagas de viento; Fenómenos Electromagnéticos

\begin{abstract}
:
World energy matrix is changing due to the increase of energy consumption in the last years. Installation of unconventional renewable energy plants in many countries has become a solution to this event. Integration of this type of energy to the electric network causes interest in the study of phenomena that affects energy production. This document is a review of concepts and events occurring on energy quality and how they interact in a wind farm. The efficiency of wind turbines is affected by many factors, the most outstanding are: substations activities and weather issues such as wind gusts, which causes variations in the power curve. Constant monitoring on electric systems and data processing offers a high potential in improving the process of production and distribution of energy.
\end{abstract}

Keywords: Quality Power; Wind Farms; Wind Gusts; Electromagnetic Phenomena

\section{Introducción}

La energía eléctrica puede ser considerada como uno de los principales insumos que mueven el mundo industrial. Sin ella las empresas se detendrían y las economías enteras se paralizarían. Debido a su importancia es sumamente necesario saber administrarla.

Se ha analizado que más de la mitad de la energía eléctrica que se produce en el mundo es consumida por los sectores industriales y comerciales (Ramírez \& Cano, 2006) por lo que es

\footnotetext{
${ }^{1}$ Instituto de Eficiencia Energética y Energías Renovables, Coordinación General Técnica, Quito - Ecuador (gonzalo.guerron@iner.gob.ec, edwin.garcia@iner.gob.ec, andres.montero@iner.gob.ec)
} 
necesario dar un buen uso a este tipo de energía, siendo el ahorro de energía una de las mejores opciones en la reducción de costos de operación y con lo que se logra mejoras en los estándares de competitividad dentro del mundo industrial.

Uno de los aspectos que ha tomado gran importancia con la integración de los diferentes sistemas eléctricos, es el estudio de la calidad de energía enfocado a la generación de electricidad con fuentes renovables no convencionales, como es el caso de la energía eólica. (Llamas \& De los Reyes, 2010). Debido a que esta energía renovable es una de las más utilizadas en la generación eléctrica de alta potencia, es importante contar con indicadores de eficiencia energética para mantener el equilibrio entre la calidad de energía y su producción.

La calidad de energía se refiere a varios tipos de perturbaciones eléctricas como: elevaciones y caídas de voltaje, parpadeos, impulsos, armónicos, componentes de voltaje continuo, transitorios, interferencia electromagnética e interrupciones en el sistema eléctrico; también se puede clasificar este tipo de perturbaciones en tres grupos de variaciones: frecuencia, amplitud y fenómenos transitorios (Ozgonenel et al., 2013). En el caso de parques eólicos, la calidad de energía se ve afectada por los cambios bruscos en la velocidad del viento llamados ráfagas, las cuales disminuyen el rendimiento de los aerogeneradores. Este problema da como resultado un punto de partida para futuras investigaciones.

\section{Metodología}

En este trabajo se revisaron algunas de las variables que influyen en la calidad de energía, generada por los parques eólicos; para ello se realizó una recopilación bibliográfica acerca de las posibles perturbaciones provocadas: en la operación de un parque, condiciones atmosféricas como ráfagas de viento y factores externos.

\subsection{Calidad de Energía}

La calidad de energía se conoce como un conjunto limitado de propiedades eléctricas que permite a todos los sistemas eléctricos funcionar de la manera prevista sin pérdidas significativas de rendimiento y de la vida útil de los equipos.

El término se utiliza para describir a la energía eléctrica que alimenta una carga y la capacidad de ésta para que funcione correctamente, tomando en cuenta esto, se puede decir que sin la adecuada potencia los dispositivos eléctricos pueden funcionar de manera incorrecta 0 simplemente no funcionar en lo absoluto (Saini \& Kapoor, 2012).

Se considera un problema de calidad de energía eléctrica cuando existen perturbaciones ambientales en el sistema interconectado de redes eléctricas como descargas atmosféricas y cargas no lineales (Ozgonenel et al., 2013). Estas perturbaciones se producen en el orden de los microsegundos y pueden causar desviación de tensión, de corriente o de frecuencia, provocando 
un mal funcionamiento en los dispositivos de uso final como: pérdida de datos, funcionamiento errático de controles electrónicos, pérdidas en producción empresarial y deterioro de la economía o el bienestar de los usuarios finales.

\subsection{Importancia actual}

En la actualidad, el enfoque de los problemas en calidad de energía se ha convertido en uno de los temas más importantes en los sistemas de generación debido a la rápida industrialización y al uso extensivo de cargas no lineales que provocan distorsión a la red de corriente alterna (Arya \& Singh, 2014).

De acuerdo a normativas internacionales, se debe realizar un nuevo diseño para los dispositivos de detección de calidad de energía basado en los sistemas embebidos industriales que poseen rápida velocidad de cálculo, alta tasa de almacenamiento, precisión y una gran estabilidad incondicional en el sistema de control. Mediante estos controles se obtienen beneficios económicos para las empresas, logrando un aumento en la competitividad y confiabilidad, mediante la optimización de su proceso productivo (Junfeng et al., 2012).

Por otro lado el aumento de los equipos de automatización incrementan los problemas de confiabilidad en las líneas de producción, siendo estos equipos electrónicos una fuente de perturbaciones para la calidad de energía eléctrica debido a su producción de distorsiones en las ondas de tensión y corriente (De Battista, 2000).

Tomando en cuenta lo anterior, se han desarrollado sistemas inteligentes de monitoreo de redes eléctricas que permiten resolver problemas como: huecos de tensión, apagones, sobrecargas, redes obsoletas, etc. (Fadaeenejad et al., 2014).

\subsection{Características de los fenómenos electromagnéticos}

A continuación se describen los fenómenos electromagnéticos que afectan a un punto determinado de la red eléctrica. La Tabla 1 presenta los fenómenos en régimen permanente, considerando las caídas de voltaje, elevaciones de voltaje, bajo voltaje y sobre voltaje.

En la Tabla 2 se pueden ver la descripción de los fenómenos en régimen transitorio: interrupciones, armónicos, flickers, ruido y transitorios. 
Tabla 1. Fenómenos electromagnéticos en régimen permanente

\begin{tabular}{|c|c|c|}
\hline Fenómeno & Descripción & Figura \\
\hline $\begin{array}{c}\text { Caída de } \\
\text { voltaje }\end{array}$ & $\begin{array}{l}\text { Las caídas de voltaje son los fenómenos comunes } \\
\text { en los últimos años (SAG o DIP en inglés). Se define } \\
\text { como una reducción en el nivel normal de tensión } \\
\text { entre el } 10 \text { y } 90 \% \text { del voltaje p.u con una duración } \\
\text { aproximada de } 0.5 \text { ciclos a } 1 \text { minuto (Almeida et al., } \\
\text { 1992). } \\
\text { Estos fenómenos pueden ser causados por la } \\
\text { conexión o puesta en servicio de grandes cargas, } \\
\text { arranque de motores o por fallas en el sistema de } \\
\text { distribución o transmisión en la red. Los daños } \\
\text { causados por estas caídas de voltaje están } \\
\text { relacionados principalmente a procesos } \\
\text { interrumpidos, los cuales pueden ocasionar pérdidas } \\
\text { económicas altas en el sector industrial (Barrantes, } \\
\text { 2007) (Fischer, 1989). }\end{array}$ & $\begin{array}{l}\prod_{\text {Figura 1. Caída de voltaje }} \\
\text { Fuente: (Ramírez \& Cano, } \\
\text { 2006) }\end{array}$ \\
\hline $\begin{array}{c}\text { Elevaciones de } \\
\text { voltaje }\end{array}$ & $\begin{array}{l}\text { Estos fenómenos son menos frecuentes que las } \\
\text { caídas de voltaje, son incrementos momentáneos en } \\
\text { el valor efectivo de voltaje p.u. y están fuera de las } \\
\text { tolerancias normales de cada sistema del valor } \\
\text { nominal. Pueden durar de medio ciclo hasta } 1 \\
\text { minuto. Generalmente son causados al conectar y } \\
\text { desconectar grandes cargas o bancos de } \\
\text { condensadores (Barrantes, 2007). }\end{array}$ & $\begin{array}{l}\text { Figura 2. Elevaciones de voltaje } \\
\text { Fuente: Adaptado (Ramírez \& } \\
\text { Cano, 2006) }\end{array}$ \\
\hline Bajo voltaje & $\begin{array}{l}\text { Las caídas de voltaje son fenómenos comunes en } \\
\text { los últimos años. Éstas se definen como una } \\
\text { reducción en el nivel normal de tensión entre el } 10 \text { y } \\
90 \% \text { del voltaje. Su duración es de más de un minuto } \\
\text { (Barrantes, 2007). }\end{array}$ & $\begin{array}{l}\text { Figura 3. Bajo voltaje } \\
\text { Fuente: Adaptado de (Ramírez } \\
\text { \& Cano, 2006) }\end{array}$ \\
\hline Sobrevoltaje & $\begin{array}{l}\text { Es un incremento del voltaje por encima de la } \\
\text { tolerancia del valor p.u. sobre el } 110 \% \text { del valor } \\
\text { nominal y dura más de un minuto. (Almeida et al., } \\
\text { 1992) }\end{array}$ & $\begin{array}{l}\text { Figura 4. Sobre voltaje } \\
\text { Fuente: Adaptado de (Ramírez } \\
\text { \& Cano, 2006) }\end{array}$ \\
\hline
\end{tabular}

Tabla 2. Fenómenos electromagnéticos en régimen transitorio

\begin{tabular}{|c|l|c|}
\hline Fenómeno & \multicolumn{1}{|c|}{ Descripción } & \multicolumn{1}{c|}{ Figura } \\
\hline Interrupciones & $\begin{array}{l}\text { Las interrupciones son clasificadas según su } \\
\text { duración en: } \\
\text { Cortas o momentáneas }\end{array}$ \\
Enfoque UTE, V.5-N.3, Sep.2014, pp.25-44
\end{tabular}




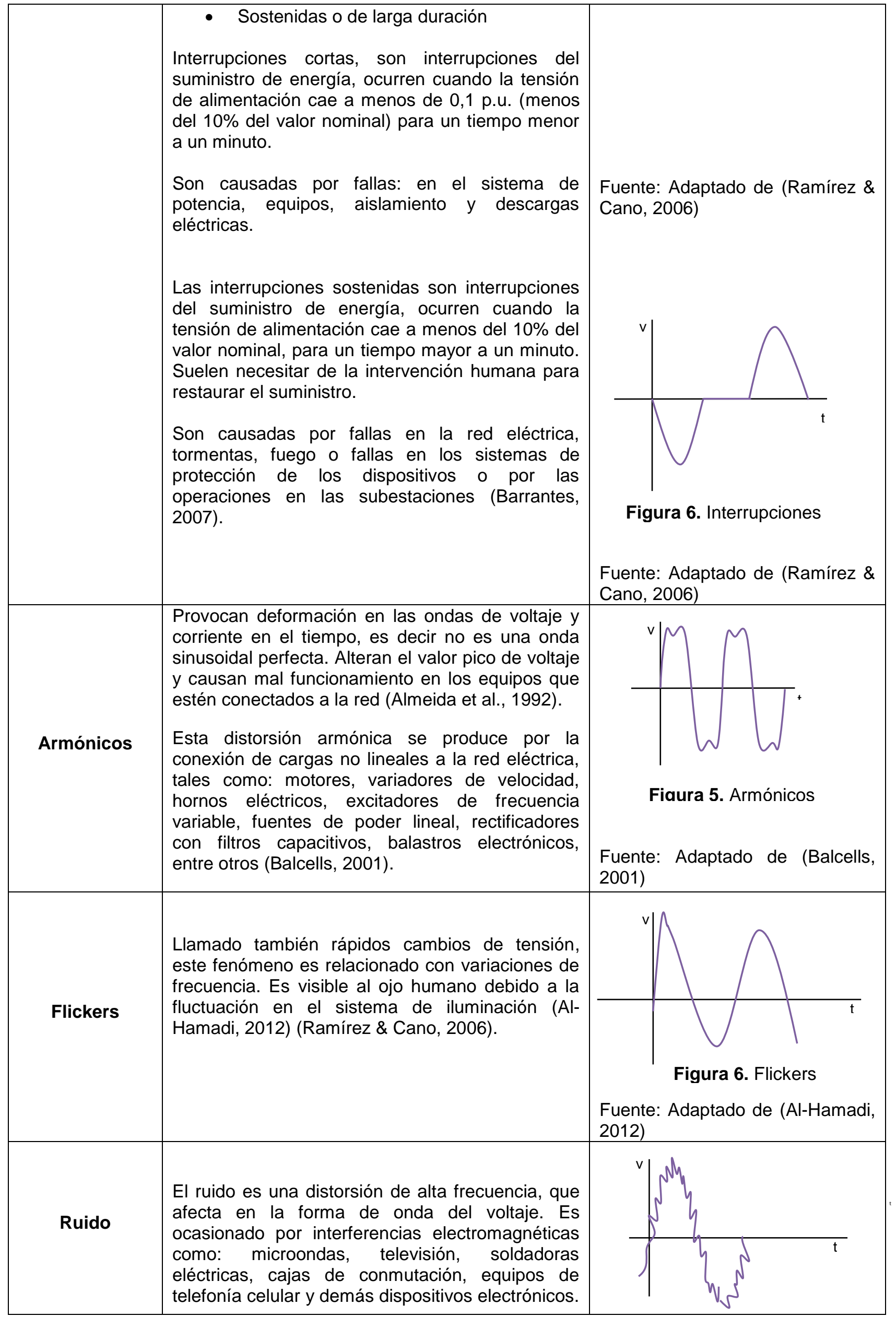




\begin{tabular}{|c|c|c|}
\hline & $\begin{array}{l}\text { Los daños que provoca el ruido son } \\
\text { principalmente pérdidas de información y errores } \\
\text { de procesamiento de datos (Ramírez \& Cano, } \\
\text { 2006). }\end{array}$ & $\begin{array}{l}\text { Figura 7. Ruido } \\
\text { Fuente: Adaptado de (Cidras \& } \\
\text { Carrillo, 2010) }\end{array}$ \\
\hline Transitorios & $\begin{array}{l}\text { Un transitorio es un cambio en la forma de onda } \\
\text { sinusoidal y su duración está en el rango desde } \\
\text { los microsegundos hasta } 5 \text { milisegundos. (La \& } \\
\text { La, 2010.) } \\
\text { Se los clasifica en dos tipos de Transitorios: de } \\
\text { impulso y oscilatorios. } \\
\text { Transitorio por impulso, es una rápida } \\
\text { elevación con suave caída, tiene un rango } \\
\text { de duración en los microsegundos. Se le } \\
\text { Ilama pico si se adiciona a la onda seno. } \\
\text { - } \\
\text { Transitorio oscilatorio, produce una } \\
\text { elevación rápida pero cae con } \\
\text { oscilaciones exponenciales, cuyos valores } \\
\text { cambian rápidamente de polaridad. Puede } \\
\text { durar un ciclo o más, su frecuencia puede } \\
\text { variar entre (500 KHz - } 5 \mathrm{MHz} \text { ). (Melhorn } \\
\text { \& McGranaghan, 1995) }\end{array}$ & $\begin{array}{l}\text { Figura 8. Transitorio por impulso } \\
\text { al., 1992). } \\
\text { v| Adaptado de (Almeida et } \\
\text { Fuente: Adaptado de (Barrantes, } \\
\text { 2007) }\end{array}$ \\
\hline
\end{tabular}

\subsection{Calidad de energía en parques eólicos}

Debido al creciente incremento de la energía eólica, el análisis y la modelación de los parques eólicos son esenciales para la estabilidad y la respuesta dinámica de los sistemas de energía. Los parques eólicos tienen diferentes impactos y funciones en el rendimiento de los sistemas eléctricos debido a la variación de la velocidad del viento en el tiempo. Se han utilizado diferentes técnicas y modelos para la determinación de estos problemas, y los impactos del parque eólico en la calidad de energía y en las redes de distribución (Mutlu et al., 2009).

En la actualidad, un número cada vez mayor de parques eólicos de pequeña potencia se utilizan como fuentes de generación distribuida y estas se conectan a los sistemas de distribución generales de cada país. La naturaleza intermitente y volátil de generación de energía eólica puede 
afectar los voltajes del sistema de distribución, frecuencia y adecuación de la generación, por lo que los parámetros eléctricos de la red de distribución tienen que ser mantenidos.

Cuando la penetración de la energía eólica es alta, el control de voltaje en el sistema de distribución se vuelve particularmente importante (Chavan, 2013). En muchos países, los nuevos códigos de red establecidos exigen que los parques eólicos incorporen generadores de inducción doblemente alimentados (DFIG) para que participar activamente en la mejora de control de voltaje en el sistema de distribución (Zhao et al., 2010).

Las investigaciones en análisis de calidad de energía en parques eólicos presentan novedosos controles, con el propósito de mitigar las emisiones de parpadeo (flickers) de los generadores de inducción doblemente alimentados. La estrategia de control que presentan se basa en un sistema de control que está compuesto por dos controladores en cascada, un control de parques eólicos y un controlador en la turbina de viento local (Perera et al., 2014), (Bystryk \& Sullivan, 2011).

El control del parque eólico se adapta a los consumos de la red de distribución y genera la potencia reactiva mínima que permite suavizar las variaciones rápidas de la producción de energía eólica. En este sentido, el control del parque eólico facilita la integración en las redes eléctricas débiles mediante el intercambio de potencia reactiva con la red. Por otra parte, este tipo de controlador posee las características para dar estabilidad, rapidez y robustez en el despacho del parque eólico, este tipo de control garantiza que la turbina de viento local pueda cambiar y controlar el punto de ajuste de la potencia reactiva con la red (Girbau et al., 2014).

Para lograr una mejor respuesta del parque se utiliza la teoría del control robusto, la cual maneja el diseño de sistemas de control dinámicos que poseen incertidumbres en sus modelos, se dice que un sistema es robusto para un determinado conjunto de incertidumbres si proporciona solidez y un adecuado rendimiento para los modelos integrales del sistema. De esta forma podemos disminuir las perturbaciones del sistema y conectar hacia el sistema interconectado la producción de energía de las turbinas de viento (Olamaei et al., 2012), (Boutoubat et al., 2013).

En las turbinas de viento, los parpadeos pueden ser causados por operaciones de conmutación y por las fluctuaciones de la potencia activa y / o reactiva en la operación continua (Attya \& Hartkopf, 2013)

Las mediciones de flicker según la norma 61400-21 deben ser realizadas, en una simulación en una red aislada al sistema nacional interconectado, con el fin de poder determinar las características del fenómeno (Tascikaraoglu et al., 2011).

Las centrales eólicas modernas tales como generadores de inducción doblemente alimentados (DFIGs) han demostrado capacidades de potencia reactiva que les permiten operar en diversos 
modos como: el control de factor de potencia y la operación de control de tensión (Fadaeenejad et al., 2014), (Mansouri et al., 2004).

En el futuro, será necesario utilizar diferentes estrategias de control con el fin de prestar servicios auxiliares tales como: soporte de potencia reactiva y control en el voltaje del sistema a la red de energía, existen escasos estudios sobre el impacto en la emisión de parpadeo cuando las centrales de energía eólica están operando bajo este tipo de estrategias de control, solo existe un conocimiento global sobre la propagación de parpadeo y atenuación en las redes, esencialmente relacionada con cargas fluctuantes, donde los flujos de potencia activa y reactiva son unidireccionales, es decir, aguas arriba a aguas abajo (Gupta et al., 2014).

En las redes de distribución con la generación integrada, el flujo de potencia activa y reactiva podría llegar a ser bidireccional. Por otra parte, las cargas conectadas en este tipo de redes también pueden influir en la propagación de parpadeo y atenuación (Al-Hamadi, 2012).

Al conectar un parque eólico a la red se producen distorsiones conocidas como armónicos que pueden afectar en la potencia activa, esto se debe principalmente a la utilización de conversores de onda AC a DC y de DC a AC en las turbinas eólicas (Arya \& Singh, 2014). Con el fin de mitigar la generación de armónicos se utiliza filtros en el sistema de conversión de onda de la máquina eólica, además un sistema de monitoreo a la salida de cada transformador permite realizar un estudio de la variación de la onda de corriente y voltaje (Jadán \& Calle, 2009).

Los armónicos se presentan independientemente de la capacidad de producción que tenga el o los aerogeneradores conectados a la red, sin embargo tienden a aumentar debido a interarmónicos que caracterizan a los armónicos. Al incrementar la producción de potencia de un aerogenerador, el total de interarmonicos también aumenta. Los armónicos no dependen de la potencia activa de producción, mientras que los interarmonicos tienen una relación de dependencia con la potencia activa de producción (Yang et al., 2014), (Yeh \& Huang, 2014).

\subsection{Ráfagas de viento}

La calidad de energía en generadores eólicos se ve afectada por el comportamiento y características del viento. Mediante el modelamiento de ráfagas frontales de viento, aplicado al comportamiento de las diferentes estructuras de un aerogenerador, se determina que los efectos dinámicos en estos tienen una mayor frecuencia de influencia con relación a los efectos cinemáticos causados por las diferentes capas de viento (Kun et al., 2012), (Morsi \& El-Hawary, 2011).

Los modelos de ráfaga de viento se basan en la norma IEC-TC88 (Cheng \& Bierbooms, 2001), en la que se establecen propiedades estocásticas para forma y duración de la ráfaga; por lo tanto con 
una simulación sobre un aerogenerador se obtiene una estimación mínima y máxima de operación y control (Dicorato et al., 2012).

\subsection{Generador sincrónico bajo influencia de una ráfaga}

Para el estudio de una turbina eólica que incluye un generador sincrónico con imanes permanentes, se determinó su comportamiento utilizando el modelo matemático Skystream (Li \& Li, 2012), (Cultura \& Ziyad, 2012), y para la simulación de la ráfaga de viento se utilizó la función escalón, con lo cual visualiza el comportamiento de la potencia en función de la corriente y voltaje en la salida del generador.

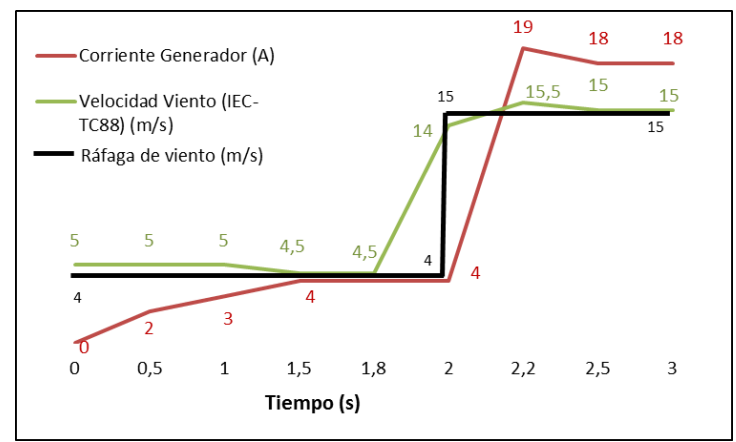

Figura 12. Simulación de un generador sincrónico ante una ráfaga de viento Fuente: Modificado de (Cultura \& Ziyad, 2012)

En la Figura 12 se observa que la velocidad del viento es constante en $4 \mathrm{~m} / \mathrm{s}$ y al introducir la función escalón, ésta se incrementa a $15 \mathrm{~m} / \mathrm{s}$, la corriente aumenta linealmente de 4 a 19 amperios en 0,2 segundos y se estabiliza en 18 amperios en un periodo de 1 segundo; el voltaje de salida en el generador permanece constate debido al inversor instalado en este tipo de dispositivos.

Las pérdidas generadas por el efecto de las ráfagas de viento, crean un impacto significativo en la potencia de salida de los aerogeneradores, afectando la producción total de las turbinas de viento; el incremento de pérdidas es directamente proporcional con la turbulencia del viento en turbinas de baja potencia (Kuenzel \& Bikash, 2014). Las simulaciones de la curva de potencia se basan en la norma IEC 61400-12-1, donde se desprecia el impacto de la turbulencia sobre las turbinas (Lubitz, 2014), (Pourrajabian et al., 2014).

\subsection{Influencia de las ráfagas de viento sobre generadores de inducción doblemente alimentados}

El parque eólico en Golfo El Zayt (Egipto) tiene una potencia instalada de 120 MW con generadores de inducción doblemente alimentados (DFIG). El funcionamiento de estas máquinas se basa en: "el giro del rotor a una frecuencia diferente que la frecuencia de red" (Noureldeen \& Rashad, 2013), obteniendo la máxima potencia frente a una velocidad variable del viento, logrando controlar la potencia activa y reactiva de forma independiente. 
Noureldeen y Rashad realizaron una simulación del parque Zayt y analizaron la estabilidad para diferentes condiciones de ráfagas de viento. La variación de la velocidad de la ráfaga se simuló en tres diferentes condiciones de viento; las velocidades promedio son de 1112 y $13 \mathrm{~m} / \mathrm{s}$; el valor nominal en esta simulación es de $12 \mathrm{~m} / \mathrm{s}$. Como se muestran en la Figura 13. Las ráfagas de viento tendrán un pico máximo de 6 m/s sobre las velocidades (Noureldeen \& Rashad, 2013).

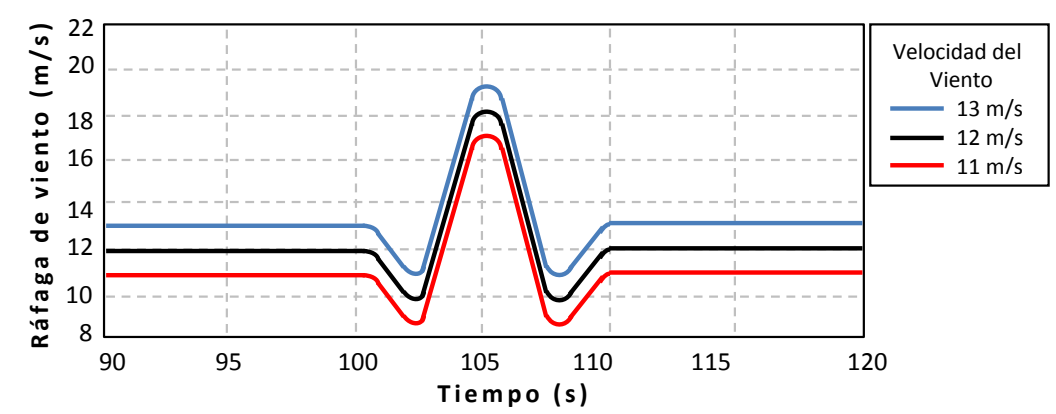

Figura 13. Simulación del viento y presencia de una ráfaga Fuente: Modificado de (Noureldeen \& Rashad, 2013).

Esta simulación establece el comportamiento de los aerogeneradores frente a una ráfaga de viento (Figura 13), determinando la relación inversamente proporcional entre la potencia activa y la potencia reactiva acorde al incremento de la velocidad del viento, como se muestra en la Figura 14.

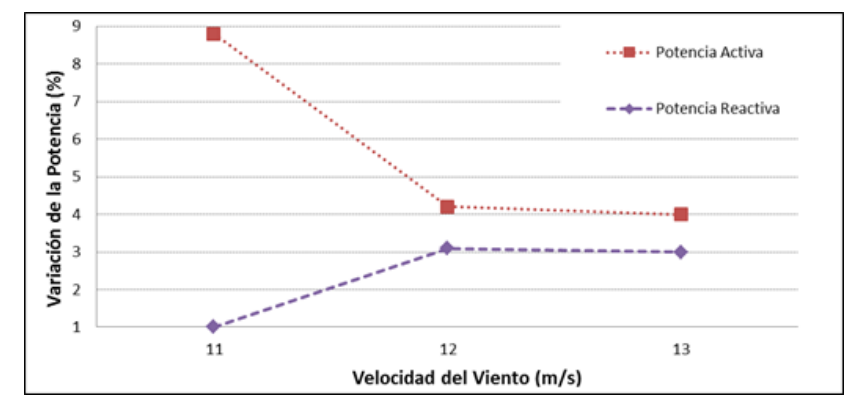

Figura 14. Variación de la potencia active y reactiva frente a una ráfaga Fuente: Modificado de (Noureldeen \& Rashad, 2013).

\subsection{Cortocircuitos en aerogeneradores en presencia de ráfagas de viento}

El cortocircuito en parques eólicos puede presentarse cuando una ráfaga de viento atraviesa el aerogenerador ocasionando inestabilidad y disminuyendo la confiabilidad del parque; las protecciones eléctricas juegan un rol importante en la estabilidad del sistema al realizar la desconexión del sistema eléctrico (Rocha, 2011).

En un estudio realizado en el parque eólico en las islas de Cabo Verde se determinó el comportamiento de las potencias de un aerogenerador ante el corto circuito producido por una ráfaga de viento (Teixeira et al., 2011), (Zeng et al., 2012). El perfil del comportamiento de la velocidad de viento se basa en la recolección de datos de las diferentes estaciones 
meteorológicas instaladas del parque; la simulación se basa en la introducción de una ráfaga de viento sobre una velocidad promedio de $5 \mathrm{~m} / \mathrm{s}$, alcanzando una velocidad máxima de $20 \mathrm{~m} / \mathrm{s}$ como se muestra en la Figura 14.

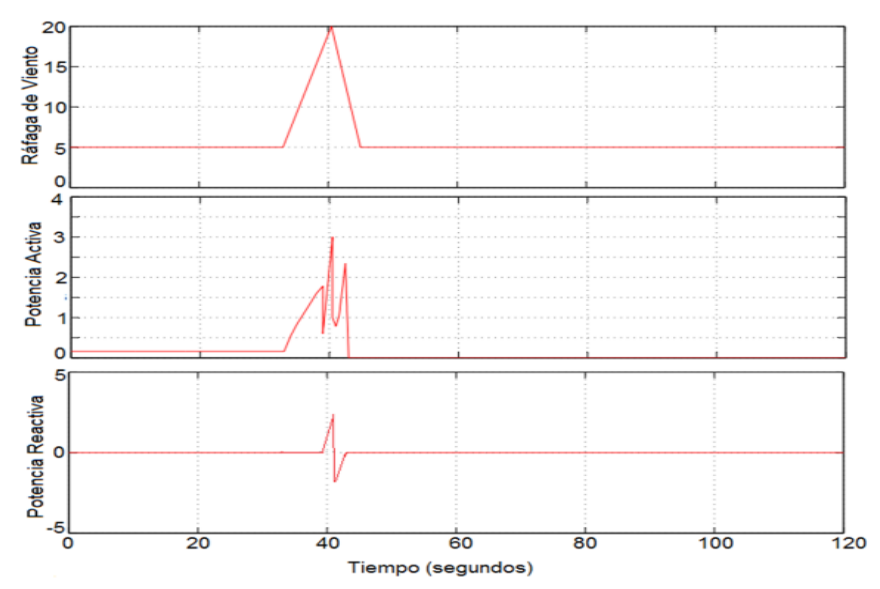

Figura 15. Modelo de ráfaga de viento.

Fuente: Modificado de (Teixeira et al., 2011).

La respuesta obtenida de la simulación determina que la potencia activa sufre un incremento con respecto a la potencia nominal por presencia de la ráfaga de viento, la potencia reactiva permanece constante; en el instante en el que se presenta un corto circuito las potencias sufren oscilaciones, actuando las protecciones y despejando la falla, como se muestra en la Figura 15.

\subsection{Fenómenos de régimen permanente en parques eólicos}

Para analizar la calidad de energía en generadores eólicos se analiza el comportamiento de algunos fenómenos en régimen transitorio como: armónicos, flicker, ferroresonancia, agujeros de tensión, micro-cortes y transitorios de conexión.

\subsubsection{Armónicos}

Los aerogeneradores no están exentos de tener distorsión armónica de voltaje y corriente, en estos dispositivos el fenómeno se presenta por diversos aspectos tales como: saturación en los transformadores, operaciones en la subestaciones, conexiones de cargas no lineales a la red entre otros (Kesraoui et al., 2014).

Los aerogeneradores dependen del comportamiento del viento, para mantener constante el voltaje en la salida se utilizan inversores, que producen armónicos por la operación de los tiristores; estos equipos disponen de un sistema de filtros para controlar y suprimir estos niveles de armónicos, logrando controlar la frecuencia natural (Gimenez \& Gómez, 2011), (De Battista, 2000).

Los armónicos se representan por la distorsión armónica total (THD), y están estandarizados bajo la norma IEC 61000-3-6 (Kesraoui et al., 2014). En la Figura 16 se muestra la distorsión que generan los armónicos sobre la onda de voltaje que entrega el aerogenerador (Arcila, 2012). 


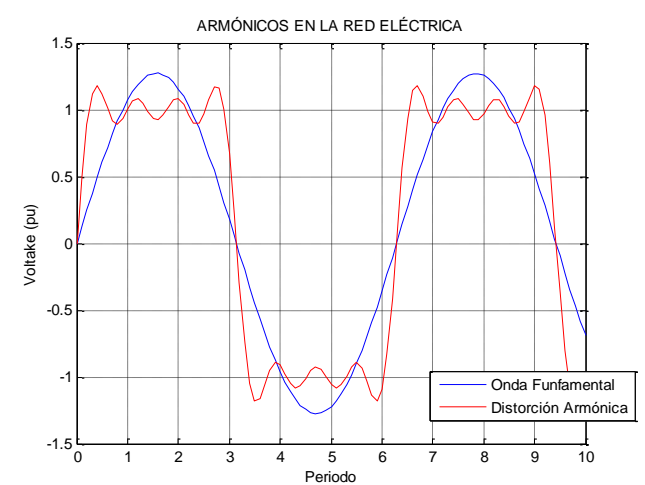

Figura 16. Armónicos en la Red

Fuente: Modificada de (Kesraoui et al., 2014)

\subsubsection{Flicker}

Analizando la presencia de flickers en aerogeneradores, se determinó que la distorsión es provocada a la conexión de una máquina eléctrica en la red, generando sobre corriente a la red de corta duración. La estandarización de los flicker fue realizada por la Comisión Electrotécnica Internacional (IEC) descrita en la norma IEC 61000-4-15 (Ignacio, 2010), (Chavan, 2013).

Los flickers de régimen permanente presentes en turbinas eólicas, son causados por ráfagas de viento variando la velocidad en los aerogeneradores, por lo tanto este fenómeno no puede ser compensado ni controlado en su totalidad por el sistema de control, como se muestra en la Figura 17. La mitigación de flickers mecánicamente se puede realizar con el incremento en el diámetro de las palas (Girbau et al., 2014).

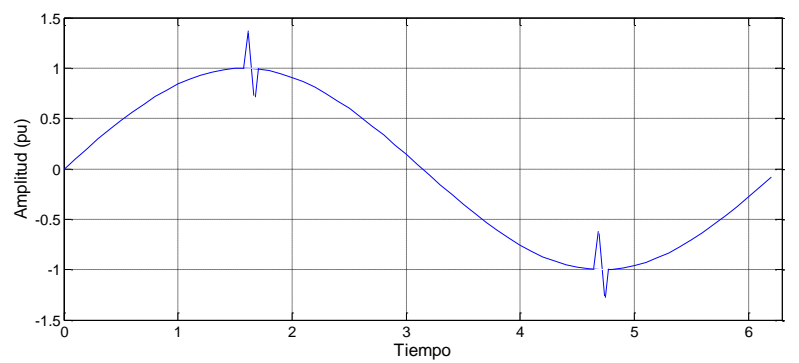

Figura 17. Fluctuación voltaje sinusoidal Fuente: Modificada de (Ignacio, 2010).

\subsubsection{Ferroresonancia}

En los parques eólicos existe la necesidad de elevar el voltaje a los niveles de los sistemas eléctricos interconectados, esto ocasiona la utilización de transformadores elevadores de voltaje a través de una conexión estrella-triangulo como se muestra en la Figura 18 (Gimenez \& Gómez, 2011).

El fenómeno de ferroresonancia es el resultado de la capacitancia parasita de los cables aguas abajo del transformador y de su inductancia primaria, afectando a la excitación de un transformador o de inductancias, mediante la conexión en serie de capacitancias; esto se 
encuentra especificado en la norma ANSI/IEEE Std 100-1984 (Hassan et al., 2011) (Kuenzel \& Bikash, 2014).

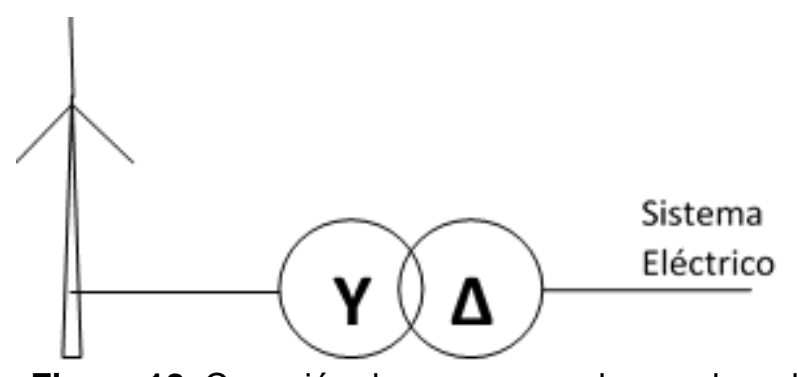

Figura 18. Conexión de aerogeneradores a la red

\subsection{Fenómenos de régimen transitorio}

\subsubsection{Huecos de tensión}

Los huecos de tensión son fenómenos transitorios que se presentan en todos los tipos de generación eléctrica y en la industria eólica no es la excepción. Este fenómeno se caracteriza por una reducción brusca de la tensión y un tiempo de recuperación como se muestra en la Figura 19. En un parque eólico se puede presentar después del paso de una ráfaga de viento (Gimenez \& Gómez, 2011).

Los huecos de tensión se presentan en una o dos de las fases, generan oscilaciones en el rotor de la turbina causando el paro abrupto del aerogenerador (Jiménez, Gómez-Lázaro, Fuentes, Molina-García, \& Vigueras-Rodríguez, 2013).

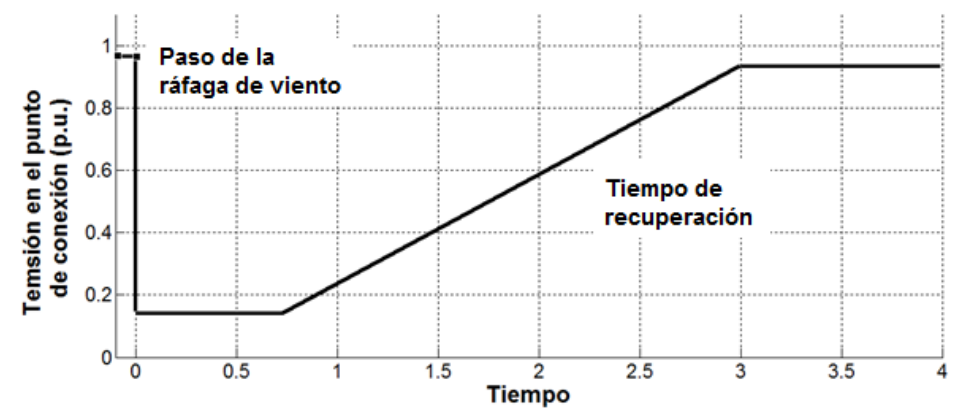

Figura 19. Huecos de voltaje en generación de electricidad mediante turbinas eólicas.

Fuente: Modificada de (Gimenez \& Gómez, 2011)

\subsubsection{Transitorios de conexión}

\section{Micro cortes}

Los micro cortes de voltaje son fenómenos transitorios que se generan por la velocidad de operación de los dispositivos de maniobra como por ejemplo interruptores y re-conectadores en la subestación de la central eólica, los cuales generan picos o huecos de tensión con una duración de micros segundos como se muestra en la Figura 20; (Gimenez \& Gómez, 2011), estos afectan a la calidad de energía causando deformidad de la onda. 
Existen otros fenómenos transitorios que afectan a los parques eólicos como:

- Corriente de cortocircuito: Se caracteriza por sus perturbaciones al presentarse una falla en el sistema.

- Sobre-intensidad: Se genera en la red por conexión del generador eólico.

- Compensación capacitiva: Se presenta cuando se mejora el factor de potencia del parque eólico (Gimenez \& Gómez, 2011).

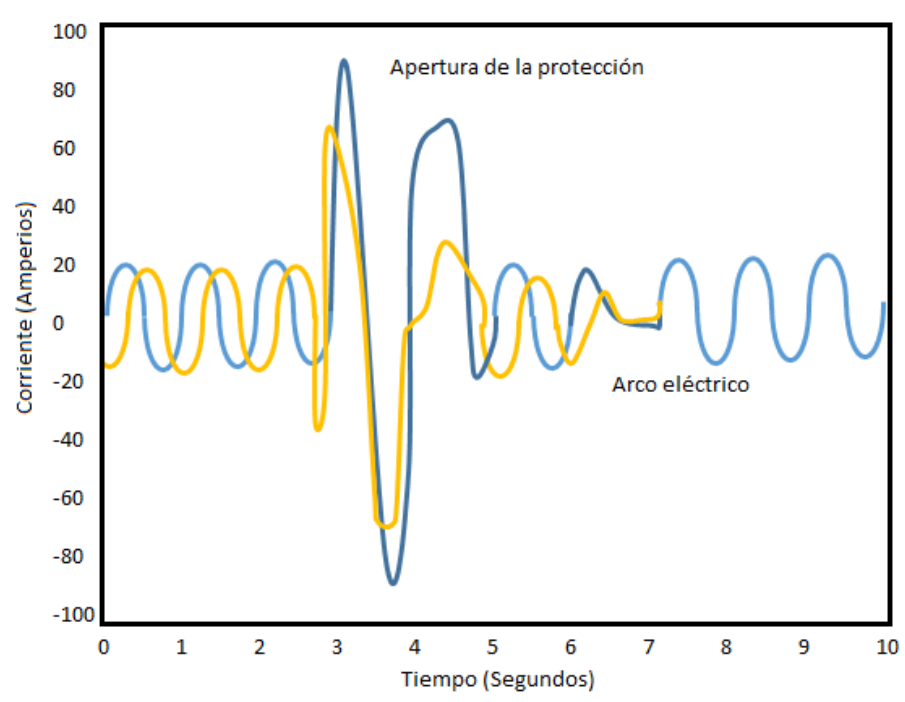

Figura 20. Forma de onda de Sobre-corriente

Fuente: Modificada de (Gimenez \& Gómez, 2011)

\section{Discusión}

La eficiencia de los parques eólicos se ve afectada por diversos factores entre los más significativos se destacan: maniobras en subestaciones y factores climáticos; la generación de energía en un parque eólico no es constante, esto influye directamente en la calidad de energía reduciendo la eficiencia.

Es oportuno realizar un estudio de calidad de energía en los parques eólicos, con el fin de verificar el correcto comportamiento del parque y obtener resultados que puedan ser utilizados como referencias en próximas centrales eólicas a instalarse.

Es necesario realizar un análisis de calidad de energía en parques eólicos que presenten condiciones atípicas de instalación como los parques instalados sobre los 2200 msnm.

\section{Conclusiones}

Existen pocos estudios de calidad de energía realizados en parques eólicos. En muchos casos los resultados no fueron utilizados, por lo que es importante realizar nuevas auditorías con sus respectivas aplicaciones prácticas. 
La calidad de energía en parques eólicos se ha convertido en un tema de gran interés para muchos investigadores por dos factores: el económico al ser una de las fuentes de energía renovable con más aceptación que las demás y por la variabilidad que existe en la potencia al estar directamente relacionada con el viento.

El monitoreo constante de la calidad de energía en los sistemas eléctricos y el análisis minucioso de estos datos, ofrece un alto potencial para mejorar los procesos de producción y distribución de la energía.

Los análisis de calidad de energía permiten desarrollar mapas de estrategias de control, evitando pérdidas económicas a niveles de transmisión y distribución mediante la actualización de estos sistemas.

Los fenómenos producidos en cuanto a calidad de energía en un parque eólico, se generan por dos causas relevantes: la variación del viento y los dispositivos de electrónica de potencia que conforman el aerogenerador. Estos fenómenos aunque no pueden ser eliminados si pueden ser controlados para que se mantengan dentro de los límites establecidos en la ley del país o de acuerdo a las normas internacionales.

El paso de una ráfaga de viento por más corta duración que presente, afecta al aerogenerador ocasionando una disminución en la calidad de energía, su estructura mecánica también se ve involucrada.

La forma más sencilla para la simulación de una ráfaga de viento en un aerogenerador es mediante el uso de la función escalón, la cual es efectiva cuando se delimitan los rangos de estudio de la velocidad. Para obtener resultados más precisos se debe implementar un modelo con datos meteorológicos y de operación del parque.

Si la ráfaga de viento supera el valor máximo de velocidad descrita en la curva de potencia del aerogenerador, causa una desconexión afectando a la calidad de energía de todo el parque.

Una ráfaga de viento no involucra a todo el parque, ésta es puntual en algunos aerogeneradores, por lo tanto la calidad de energía que se extrae de uno o varios, se suma a la de los demás en la subestación más cercana.

En granjas eólicas la ráfaga de viento influye directamente en la primera fila de los aerogeneradores, por efecto de las estelas, éstas afectan indirectamente a los aerogeneradores de las siguientes filas. 


\section{Bibliografía}

Al-Hamadi, H. M. (2012). Fuzzy logic voltage flicker estimation using Kalman filter. International Journal of Electrical Power \& Energy Systems, 36(1), 60-67.

doi:10.1016/j.jepes.2011.10.024

Almeida, A. De, Delgado, L. M. J., \& Quality, P. (1992). Power Quality Problems and New Solutions.

Arcila, J. (2012). Armónicos en sistemas eléctricos, 1-26.

Arya, S. R., \& Singh, B. (2014). Power quality improvement under nonideal AC mains in distribution system. Electric Power Systems Research, 106, 86-94. doi:10.1016/j.epsr.2013.08.008

Attya, a. B., \& Hartkopf, T. (2013). Wind farms dispatching to manage the activation of frequency support algorithms embedded in connected wind turbines. International Journal of Electrical Power \& Energy Systems, 53, 923-936. doi:10.1016/j.ijepes.2013.06.011

Balcells, J. (2001). Calidad de la red eléctrica: ¿como merdirla?, 22-26.

Barrantes, I. (2007). Elaboración de una guía práctica para la evaluación de la calidad de energía dentro de C.N.F.L.

Boutoubat, M., Mokrani, L., \& Machmoum, M. (2013). Control of a wind energy conversion system equipped by a DFIG for active power generation and power quality improvement. Renewable Energy, 50, 378-386. doi:10.1016/j.renene.2012.06.058

Bystryk, J., \& Sullivan, P. E. (2011). Small wind turbine power control in intermittent wind gusts. Journal of Wind Engineering and Industrial Aerodynamics, 99(5), 624-637. doi:10.1016/j.jweia.2011.03.001

Chavan, D. (2013). Efect of vertical winds hear on flicker in wind farm, 203-208.

Cheng, P. ., \& Bierbooms, W. A. A. . (2001). Distribution of extreme gust loads of wind turbines. Journal of Wind Engineering and Industrial Aerodynamics, 89(3-4), 309-324. doi:10.1016/S0167-6105(00)00084-2

Cidras, J., \& Carrillo, C. (2010). El fenómeno del parpadeo., 1-14. 
Cultura, A., \& Ziyad, S. (2012). Dynamic Response of a Skystream Wind Turbine to a Wind gust, 1061-1066.

De Battista, H. (2000). Control de la calidad de potencia en sistemas de conversión de energía eólica.

Dicorato, M., Forte, G., \& Trovato, M. (2012). Wind farm stability analysis in the presence of variable-speed generators. Energy, 39(1), 40-47. doi:10.1016/j.energy.2011.11.050

Fadaeenejad, M., Saberian, a. M., Fadaee, M., Radzi, M. a. M., Hizam, H., \& AbKadir, M. Z. a. (2014). The present and future of smart power grid in developing countries. Renewable and Sustainable Energy Reviews, 29, 828-834. doi:10.1016/j.rser.2013.08.072

Fischer, F. (1989). Voltage Dip-Proofing Voltage Dip-Proofing.

Gimenez, J., \& Gómez, C. (2011). Wind generation using different generators considering their impact on power system, 169, 95-104.

Girbau, F., Sumper, A., Díaz, F., \& Galceran, S. (2014). Flicker mitigation by reactive power control in wind farm with doubly fed induction generators. International Journal of Electrical Power \& Energy Systems, 55, 285-296. doi:10.1016/j.ijepes.2013.09.016

Gupta, N., Swarnkar, A., \& Niazi, K. R. (2014). Distribution network reconfiguration for power quality and reliability improvement using Genetic Algorithms. International Journal of Electrical Power \& Energy Systems, 54, 664-671. doi:10.1016/j.ijepes.2013.08.016

Hassan, S., Vaziri, M., \& Vadhva, S. (2011). Review of ferroresonance in power distribution grids. Information Reuse and Integration (IRI), 2011 IEEE International Conference on, 444 - 448.

Ignacio, A. (2010). Evaluación de la emisió de flicker causada por aerogeradores conectados a la red eléctrica Uruguaya de 150kv.

Jadán, S., \& Calle, S. (2009). Análisis de la calidad de la energía eléctric a y estudio de carga de la Universidad Politécnica Salesiana Sede Cuenca, 1-34. Retrieved from http://dspace.ups.edu.ec/handle/123456789/75

Jiménez, F., Gómez-Lázaro, E., Fuentes, J. A., Molina-García, A., \& Vigueras-Rodríguez, A. (2013). Validation of a DFIG wind turbine model submitted to two-phase voltage dips following the Spanish grid code. Renewable Energy, 57, 27-34. doi:10.1016/j.renene.2012.12.032 
Junfeng, H., Hao, S., \& Xiaolin, W. (2012). Design of Power Quality Monitor Based on Embedded Industrial Computer. Physics Procedia, 24, 63-69. doi:10.1016/j.phpro.2012.02.011

Kesraoui, M., Chaib, a., Meziane, a., \& Boulezaz, a. (2014). Using a DFIG based wind turbine for grid current harmonics filtering. Energy Conversion and Management, 78, 968-975. doi:10.1016/j.enconman.2013.07.090

Kuenzel, S., \& Bikash, P. (2014). Impact of Wakes on Wind Farm Inertial Response. IEEE TRANSACTIONS ON SUSTAINABLE ENERGY, 5(1), 237-245.

Kun, D., Kareem, A., \& Butler, K. (2012). Journal of Wind Engineering Gust-front loading effects on wind turbine tower systems. Jnl. of Wind Engineering and Industrial Aerodynamics, 104-106, 109-115. doi:10.1016/j.jweia.2012.03.030

$\mathrm{Li}, \mathrm{Y}$., \& Li, H. (2012). The Impact of Wind Characteristics on the Wind Turbine Generator. 2012 Asia-Pacific Power and Energy Engineering Conference, 1-4. doi:10.1109/APPEEC.2012.6307112

Llamas, A., \& De los Reyes, J. (2010). Instrumentación para determinar la calidad del suministro eléctrico, 52(81), 1-9.

Lubitz, W. D. (2014). Impact of ambient turbulence on performance of a small wind turbine. Renewable Energy, 61, 69-73. doi:10.1016/j.renene.2012.08.015

Mansouri, M. N., Mimouni, M. F., Benghanem, B., \& Annabi, M. (2004). Simulation model for wind turbine with asynchronous generator interconnected to the electric network. Renewable Energy, 29(3), 421-431. doi:10.1016/S0960-1481(03)00225-8

Melhorn, C. J., \& McGranaghan, M. F. (1995). Interpretation and analysis of power quality measurements. IEEE Transactions on Industry Applications, 31(6), 1363-1370. doi:10.1109/28.475727

Morsi, W. G., \& El-Hawary, M. E. (2011). Power quality evaluation in smart grids considering modern distortion in electric power systems. Electric Power Systems Research, 81(5), 11171123. doi:10.1016/j.epsr.2010.12.013

Mutlu, Ö. S., Akpınar, E., \& Balıkcı, A. (2009). Power quality analysis of wind farm connected to Alaçatı substation in Turkey. Renewable Energy, 34(5), 1312-1318. doi:10.1016/j.renene.2008.09.012 
Noureldeen, O., \& Rashad, A. (2013). Modeling and investigation of Gulf El-Zayt wind farm for stability studying during extreme gust wind occurrence. Ain Shams Engineering Journal, 5(1), 137-148. doi:10.1016/j.asej.2013.09.011

Olamaei, J., Javan, J., Yavartalab, a., \& Khederzadeh, M. (2012). Advanced Control of FACTS Devices for Improving Power Quality Regarding to Wind Farms. Energy Procedia, 14, 298303. doi:10.1016/j.egypro.2011.12.933

Ozgonenel, O., Yalcin, T., Guney, I., \& Kurt, U. (2013). A new classification for power quality events in distribution systems. Electric Power Systems Research, 95, 192-199. doi:10.1016/j.epsr.2012.09.007

Perera, D., Meegahapola, L., Perera, S., \& Ciufo, P. (2014). Characterisation of flicker emission and propagation in distribution networks with bi-directional power flows. Renewable Energy, 63, 172-180. doi:10.1016/j.renene.2013.09.007

Pourrajabian, A., Mirzaei, M., Ebrahimi, R., \& Wood, D. (2014). Effect of air density on the performance of a small wind turbine blade: A case study in Iran. Journal of Wind Engineering and Industrial Aerodynamics, 126, 1-10. doi:10.1016/j.jweia.2014.01.001

Ramírez, S., \& Cano, E. (2006). Calidad del Servicio de Energía Eléctrica.

Rocha, R. (2011). A sensorless control for a variable speed wind turbine operating at partial load. Renewable Energy, 36(1), 132-141. doi:10.1016/j.renene.2010.06.008

Saini, M. K., \& Kapoor, R. (2012). Classification of power quality events - A review. International Journal of Electrical Power \& Energy Systems, 43(1), 11-19. doi:10.1016/j.ijepes.2012.04.045

Tascikaraoglu, a., Uzunoglu, M., Vural, B., \& Erdinc, O. (2011). Power quality assessment of wind turbines and comparison with conventional legal regulations: A case study in Turkey. Applied Energy, 88(5), 1864-1872. doi:10.1016/j.apenergy.2010.12.001

Teixeira, L., Zhang, Y., \& Kang, Y. (2011). Stability analysis of power system in cape verde with wind power under wind gust and short circuit fault. 2011 4th International Conference on Electric Utility Deregulation and Restructuring and Power Technologies (DRPT), 1072-1075. doi:10.1109/DRPT.2011.5994054

Yang, K., Bollen, M. H. J., Larsson, E. O. A., \& Wahlberg, M. (2014). Measurements of harmonic emission versus active power from wind turbines. Electric Power Systems Research, 108, 304-314. doi:10.1016/j.epsr.2013.11.025 
Yeh, T.-M., \& Huang, Y.-L. (2014). Factors in determining wind farm location: Integrating GQM, fuzzy DEMATEL, and ANP. Renewable Energy, 66, 159-169.

doi:10.1016/j.renene.2013.12.003

Zeng, X., Tao, J., Zhang, P., Pan, H., \& Wang, Y. (2012). Reactive Power Optimization of Wind Farm based on Improved Genetic Algorithm. Energy Procedia, 14, 1362-1367. doi:10.1016/j.egypro.2011.12.1102

Zhao, J., Li, X., Hao, J., \& Lu, J. (2010). Reactive power control of wind farm made up with doubly fed induction generators in distribution system. Electric Power Systems Research, 80(6), 698-706. doi:10.1016/j.epsr.2009.10.036 